\title{
Identification of key miRNAs in papillary thyroid carcinoma based on data mining and bioinformatics methods
}

\author{
JIANGUO WANG, LIPING WU, YUXIA JIN, SUPING LI and XIAODAN LIU \\ Department of Prenatal Diagnostics, Jiaxing Maternity and Children Health Care Hospital, \\ Jiaxing University, Jiaxing, Zhejiang 314000, P.R. China
}

Received June 28, 2019; Accepted October 17, 2019

DOI: $10.3892 /$ br.2019.1256

\begin{abstract}
MicroRNAs (miRNAs) are a class of short (approximately 22 nucleotides), non-coding and endogenous RNA molecules that play pivotal roles in the occurrence and development of cancer. The present study aimed to investigate key miRNAs involved in papillary thyroid carcinoma (PTC). Two independent datasets (GSE73182 and GSE113629) were obtained from the GEO database. The differentially expressed miRNAs (DEmiRNAs) between PTC tissues and normal thyroid tissues were analyzed by GEO2R with the Limma R package. Key miRNAs in PTC were identified by the VennDiagram $R$ package. The targets of the key miRNAs were predicted by miRWalk and were functionally enriched by clusterProfiler $\mathrm{R}$ package. Five miRNAs including hsa-miR-146b-5p, hsa-miR15a-5p, hsa-miR-21-5p, hsa-miR-221-3p and hsa-miR-222-3p were identified as key miRNAs in PTC. The expression levels of these key miRNAs were upregulated in PTC. This finding was also confirmed in the other dataset. Target prediction of miRNAs indicated that hsa-miR-146b-5p, hsa-miR-15a-5p, hsa-miR-21-5p, hsa-miR-221-3p and hsa-miR-222-3p exhibited 2, 41, 3, 14 and 8 target genes, respectively. Enrichment analysis indicated that these key miRNAs were mainly involved in nine biological processes, such as regulation of MAP kinase activity, JNK cascade signaling and regulation of protein serine/threonine kinase activity) and in 28 pathways, including the mitogen associated protein kinase, the sphingolipid, ErbB, Ras and the C-type lectin receptor signaling pathways. In conclusion, the present study identified several key miRNAs in PTC, which serve as potential targets for PTC diagnosis and treatment.
\end{abstract}

\section{Introduction}

Papillary thyroid carcinoma (PTC) is the main histological type of thyroid cancer, accounting for $>80 \%$ of all thyroid

Correspondence to: Dr Xiaodan Liu, Department of Prenatal Diagnostics, Jiaxing Maternity and Children Health Care Hospital, Jiaxing University, 2468 East Zhong Huan Road, Nanhu, Jiaxing, Zhejiang 314000, P.R. China

E-mail: 80liuxiaodan@163.com

Key words: papillary thyroid carcinoma, microRNA, data mining, bioinformatics malignancies (1). Although most patients with PTC have low recurrence rate and exhibit optimal prognosis as determined by long term follow-up, approximately $15 \%$ of patients demonstrate aggressive behavior and poor outcome $(2,3)$. To date, the pathogenesis of PTC has not been fully identified. Therefore, detailed investigation of the biological processes involved in PTC can provide insight to the diagnosis and pathogenesis of this disease and to its potential treatment.

MicroRNAs (miRNAs) are a class of short (approximately 22 nucleotides), non-coding and endogenous RNA molecules that can suppress the expression of the corresponding protein coding genes by binding to the 3'-untranslated region (3'-UTR) of the target mRNAs $(4,5)$. Previous studies have suggested that miRNAs can function as regulators of oncogenes or of tumor suppressor genes and are involved in the occurrence and development of cancer (6-10). However, due to the limitations of the traditional biomolecular detection methods, most of the previous studies focused only on the expression and function of single miRNAs in cancer. The application of high-throughput detection technologies used for miRNA expression, such as miRNA expression microarray and miRNA sequencing has enabled the generation of an increasing number of miRNA expression profiles and their deposition in the public databases, such as Gene Expression Omnibus (GEO) and The Cancer Genome Atlas (TCGA). By analyzing these miRNA expression profiles, a comprehensive understanding of the expression levels of specific miRNAs can be attained in a biological sample at a given moment.

In the present study, high-throughput miRNA expression profiles were obtained from the GEO database (corresponding to PTC patients) and key miRNAs in PTC were subsequently identified. Furthermore, the role of specific key miRNAs in the development of PTC was further revealed by investigating the potential function of their associated target genes.

\section{Materials and methods}

miRNA expression profiles. Two independent microarray datasets (GSE73182 and GSE113629) were obtained from the GEO database (https://www.ncbi.nlm.nih.gov/geo/). GSE73182 included miRNA expression profiles of 19 primary PTCs and five normal thyroid samples, which were generated using the Agilent microarray platform (GPL20194) (11). GSE113629 included miRNA expression profiles of five pairs of PTC and 
Table I. Summary for key miRNAs in papillary thyroid carcinoma.

\begin{tabular}{llll}
\hline miRBase & \multicolumn{1}{c}{ miRBase } & \multicolumn{1}{c}{ miRBase } & \multicolumn{1}{c}{ miRNA } \\
\hline hsa-miR-221-3p & hsa-miR-221 & MIMAT0000278 & 5'-AGCUACAUUGUCUGCUGGGUUUC-3' \\
hsa-miR-146b-5p & hsa-miR-146b & MIMAT0002809 & 5'-UGAGAACUGAAUUCCAUAGGCU-3' \\
hsa-miR-222-3p & hsa-miR-222 & MIMAT0000279 & 5'-AGCUACAUCUGGCUACUGGGU-3' \\
hsa-miR-21-5p & hsa-miR-21 & MIMAT0000076 & 5'-UAGCUUAUCAGACUGAUGUUGA-3' \\
hsa-miR-15a-5p & hsa-miR-15a & MIMAT0000068 & 5'-UAGCAGCACAUAAUGGUUUGUG-3'
\end{tabular}

normal thyroid tissues, which were generated using the Agilent microarray platform (GPL24741) (12).

Data analysis. The GEO2R tool (https://www.ncbi.nlm. nih.gov/geo/geo2r/) was used to identify the differentially expressed miRNAs (DEmiRNAs) between PTC and normal thyroid tissues. Multiple-testing corrections were performed based on the Benjamini \& Hochberg method in order to correct for false-positive results. The miRNAs with the adjusted $\mathrm{P}<0.05$ and with a $\mid \log \mathrm{FCl}>1$ were selected as DEmiRNAs.

Identification of key miRNAs in PTC. TheVennDiagram $\mathrm{R}$ package was used to identify common DEmiRNAs in two independent datasets (13). These common DEmiRNAs were identified as key miRNAs involved in PTC. Furthermore, the Human Cancer Metastasis Database (HCMDB; http:// hcmdb.i-sanger.com/index) was used to analyze large-scale expression data of cancer metastasis. miRNA deep sequencing data of three PTC and three matched normal tissues was used to confirm the expression levels of the key miRNAs (14).

miRNA-target prediction. The miRWalk tool (http://mirwalk. umm.uni-heidelberg.de/) integrating three prediction tools (miRDB, TargetScan and miRTarBase) was used to predict the target genes of key miRNAs involved in PTC (15). The genes that were predicted simultaneously by the three tools were identified as miRNA target genes. The cytoscape software was used to construct the miRNA-target gene interaction networks (16).

Functional annotation. To analyze the role of key miRNAs in PTC, Gene Ontology (GO) and Kyoto Encyclopedia of Genes and Genomes (KEGG) pathway enrichment analysis were performed using ClusterProfiler package for the miRNA target genes (17). The adjusted $\mathrm{P}<0.05$ was considered to indicate statistically significant difference.

\section{Results}

Identification of DEmiRNAs. In the GSE113629 dataset 2,115 DEmiRNAs including 1,968 up- and 147 downregulated miRNAs were identified in PTC. Furthermore, the analysis indicated that 1,963 miRNAs were upregulated only in the GSE113629 dataset. In the GSE73182 dataset, seven DEmiRNAs including five up- and two downregulated miRNAs were identified in PTC. Moreover, five miRNAs (hsa-miR-146b-5p, hsa-miR-15a-5p, hsa-miR-21-5p, hsa-miR-221-3p and hsa-miR-222-3p) were upregulated in both the GSE113629 and GSE73182 datasets, which were identified as key miRNAs in PTC (Figs. 1 and 2 and Table I).

To validate the expression levels of these miRNAs in PTC, the dataset (Exp ID: EXP00227) collected by HCMDB was analyzed (Fig. 3). The results indicated that the expression levels of all these miRNAs were upregulated in PTC compared with those of the normal tissues, which was consistent with previous microarray results $(11,12)$.

Potential function of of key miRNAs. To reveal the potential function of the key miRNAs, the prediction of their target genes was initially performed by the miRWalk tool. The results indicated that hsa-miR-146b-5p exhibited two target genes (ZNRF3 and TRAF6), whereas hsa-miR-15a-5p exhibited 41 target genes (including ZNF704, SEPT2, SPTLC1, SBNO1, KATNAL1, ZBTB10, NUFIP2, TAOK1 and CRKL). hsa-miR-21-5p exhibited three target genes (PAN3, CCL1 and $M E F 2 C$ ), hsa-miR-221-3p 14 target genes (including TMCC1, PAK1, MAP3K2, MIDN, HECTD2, PIK3R1 and PCDHAC1) and hsa-miR-222-3p eight target genes (including TRPS1, PANK3, PHACTR4, DPP8, TLE3 and ZFYVE16) (Fig. 4). Subsequently, GO analysis demonstrated that the target genes of these key miRNAs were significantly enriched in nine biological processes [regulation of MAP kinase activity, Janus N-terminal kinase (JNK) cascade signaling and regulation of protein serine/threonine kinase activity] (Table II). KEGG pathway analysis indicated that the target genes of the key miRNAs identified were significantly enriched in 28 pathways, including the MAPK, the sphingolipid, the ErbB, the Ras and the C-type lectin receptor signaling pathways (Table III).

\section{Discussion}

Bioinformatic analysis for microarray and RNA-seq has been widely used recently to identify potential targets for the diagnosis and therapy of different cancer types (18-20). Xia et al (12) demonstrated that promoter DNA methylation caused silencing of miR-204 that could serve as a potential diagnostic biomarker of PTC based on miRNA expression profiling (GSE113629). Minna et al (11) highlighted that miR-451a expression was downregulated and that it targeted the protein kinase $\mathrm{B} /$ mammalian target of rapamycin pathway in PTC based on miRNA expression profiling (GSE73182). In the present study, the two sets of data were analyzed by bioinformatic methods and five key miRNAs (hsa-miR-146b-5p, hsa-miR-15a-5p, hsa-miR-21-5p, hsa-miR-221-3p and hsa-miR-222-3p) were identified in PTC. The expression levels of these key miRNAs were increased in PTC compared 

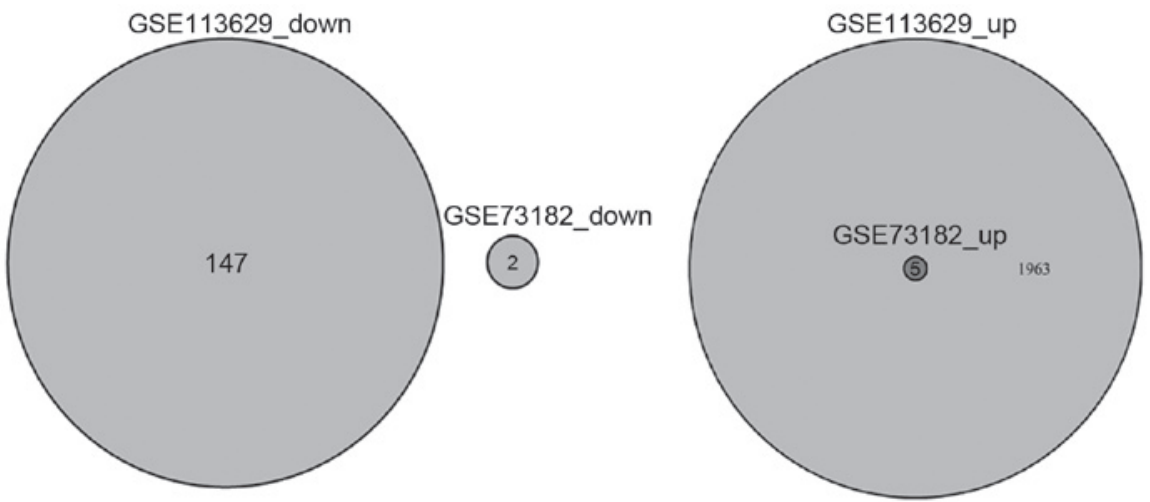

Figure 1. Venn diagrams showing the differentially expressed microRNAs in the GSE73182 and GSE113629 datasets.

GSE113629

追 Cancer 追 Normal

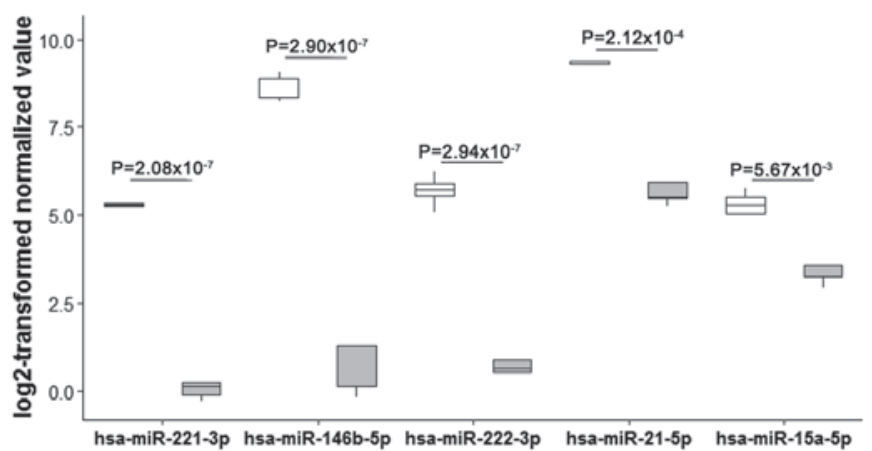

GSE73182

官 Cancer 追 Normal

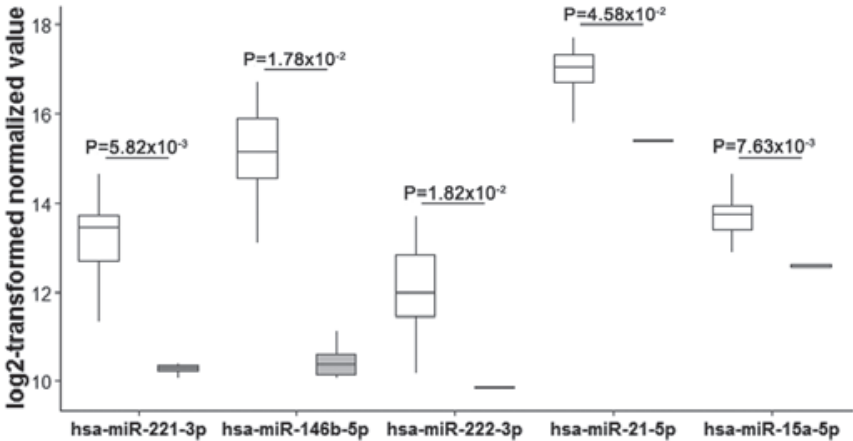

Figure 2. Boxplots indicating the expression levels of key miRNAs in papillary thyroid carcinoma based on miRNA microarray data. miRNA/miR, microRNA.
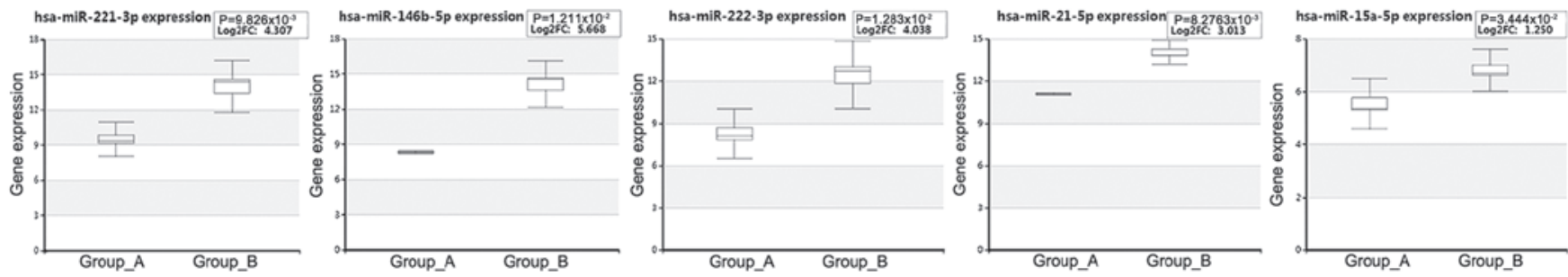

Figure 3. Boxplots indicating the expression levels of key miRNAs in PTC based on miRNA deep sequencing data (Group_A and Group_B indicated 3 normal thyroid tissues and 3 matched PTC tissues, respectively). PTC, papillary thyroid carcinoma; miRNA/miR, microRNA.

with those noted in normal tissues, which was also confirmed by an RNA-seq dataset. By reviewing the relevant literature, the data demonstrated the carcinogenic or cancer-promoting effects of hsa-miR-146b-5p and hsa-miR-222-3p in PTC. For instance, hsa-miR-146b-5p expression was associated with advanced PTC stage and promoted the development of this disease by targeting CCDC6 in vitro and in vivo, which may serve as a promising target for PTC treatment (21). The enhanced expression of miR-222-3p promoted the proliferation of PTC cells, while miR-222-3p knockdown inhibited it (22). Although no direct functional study has been conducted to date on hsa-miR-15a-5p or hsa-miR-21-5p in PTC, the findings by Jiang et al (23) and Zhang et al (24) indicated that these two miRNAs may exert tumor-suppressive effects in PTC. These results are contradictory to the present findings possibly due to tumor heterogeneity or due to the opposing function of these two miRNAs in the development of PTC. The upregulated expression of hsa-miR-221-3p in PTC has also been reported in a previous study (25). However, no relevant functional experiment was performed previously to the best of our knowledge. Therefore, further studies are required to explore the function of these key miRNAs in PTC, notably of hsa-miR-15a-5p, hsa-miR-21-5p and hsa-miR-221-3p. Chen et al (26) explored several types of RNAs involved in the development of PTC based on TCGA and identified 30 differentially expressed miRNAs between PTC and normal samples. However, hsa-miR-15a-5p, hsa-miR-21-5p, hsa-miR-221-3p and hsa-miR-222-3p were not reported in that study (26).

Considering that the miRNA function is mainly dependent on its target gene, the target genes of the key miRNAs investigated in the present study were predicted by miRWalk. 
Table II. Biological processes enrichment analysis of microRNA targets.

ID

Description

P.adjust

GO:0032147

GO:0043406

GO:0007254

GO:0071902

GO:0043507

GO:0043405

GO:0000187

GO:0071900

GO:0043506

Activation of protein kinase activity

$1.75 \times 10$

$1.85 \times 10$

Positive regulation of MAP kinase activity

$3.23 \times 10$

JNK cascade

$3.23 \times 10$

Positive regulation of protein serine/threonine kinase activity

$3.23 \times 10$

Positive regulation of JUN kinase activity

$3.23 \times 10$

Regulation of MAP kinase activity

$3.40 \times 10$

Activation of MAPK activity

$4.03 \times 10$

Regulation of protein serine/threonine kinase activity

$4.03 \times 10$

MAPK, mitogen associated protein kinase; JUN, AP-1 transcription factor subunit; JNK, Janus N-terminal kinase; GO, gene ontology.

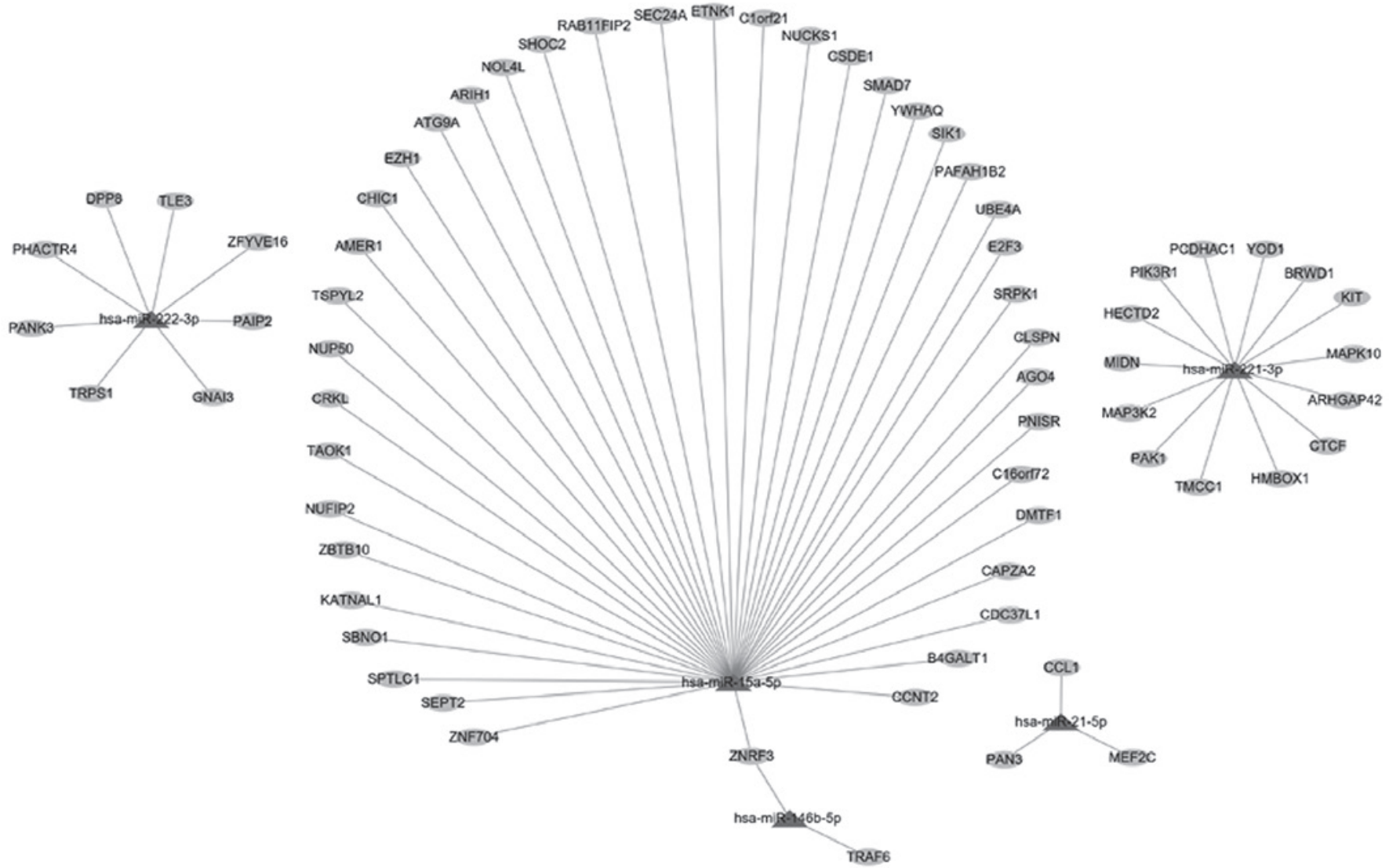

Figure 4. Network diagram showing the potential target genes of key miRNAs in papillary thyroid carcinoma. miRNA/miR, microRNA.

A total of 68 genes were identified as targets of these key miRNAs. Subsequently, GO and KEGG pathway enrichment analysis based on these target genes was performed to indicate the function of these key miRNAs in PTC. The results indicated that the key miRNAs could significantly influence nine biological processes and 28 signaling pathways, such as the regulation of mitogen associated protein kinase (MAPK) activity, JNK cascade signaling, the MAPK signaling pathway, the sphingolipid signaling pathway, the ErbB signaling pathway and the Ras signaling pathway. These observations can aid the understanding of the pathogenesis of PTC.
Although the present study identified key miRNAs in PTC based on data mining and bioinformatic methods, the following limitation must be highlighted: Functional experiments are required in future studies to elucidate the mechanism of action of the key miRNAs identified in PTC.

In conclusion, findings of the present study have revealed a group of miRNAs associated with PTC. Therefore, hsa-miR-146b-5p, hsa-miR-15a-5p, hsa-miR-21-5p, hsamiR-221-3p as well as hsa-miR-222-3p may be regarded as promising diagnostic biomarkers and therapeutic targets for PTC. 
Table III. Kyoto Encyclopedia of Genes and Genomes pathway enrichment analysis of microRNA targets.

\begin{tabular}{|c|c|c|}
\hline ID & Description & P.adjust \\
\hline hsa04010 & MAPK signaling pathway & $1.12 \times 10$ \\
\hline hsa04071 & Sphingolipid & $1.41 \times 10$ \\
\hline hsa04012 & ErbB & $2.12 \times 10$ \\
\hline hsa05170 & Human immunodeficiency virus 1 infection & 3.7810 \\
\hline hsa04014 & Ras & $4.80 \times 10$ \\
\hline hsa04140 & Autophagy - animal & $7.25 \times 10$ \\
\hline hsa04625 & C-type & $1.47 \times 10$ \\
\hline hsa05161 & Hepatitis B & $1.65 \times 10$ \\
\hline hsa05142 & Chagas & $1.66 \times 10$ \\
\hline hsa04137 & Mitophagy & $1.73 \times 10$ \\
\hline hsa05120 & Epithelial cell signaling in & $2.10 \times 10$ \\
\hline hsa04722 & Neurotrophin & $2.26 \times 10$ \\
\hline hsa05133 & Pertussis & $2.26 \times 10$ \\
\hline hsa04062 & Chemokine signaling pathway & $2.26 \times 10$ \\
\hline hsa05211 & Renal cell carcinoma & $2.35 \times 10$ \\
\hline hsa05212 & Pancreatic cancer & $2.55 \times 10$ \\
\hline hsa05418 & Fluid shear stress and atherosclerosis & $2.75 \times 10$ \\
\hline hsa04510 & Focal adhesion & $3.02 \times 10$ \\
\hline hsa04914 & Progesterone-mediated oocyte maturation & $3.23 \times 10$ \\
\hline hsa04666 & $\mathrm{Fc}$ & $3.23 \times 10$ \\
\hline hsa04024 & cAMP & $3.25 \times 10$ \\
\hline hsa00600 & Sphingolipid & $3.25 \times 10$ \\
\hline hsa04620 & Toll-like receptor signaling pathway & $3.25 \times 10$ \\
\hline hsa04912 & GnRH & $3.34 \times 10$ \\
\hline hsa04930 & Type II diabetes mellitus & $3.60 \times 10$ \\
\hline hsa01522 & Endocrine resistance & $3.70 \times 10$ \\
\hline hsa04141 & Protein processing in endoplasmic reticulum & $3.70 \times 10$ \\
\hline hsa04622 & RIG-I-like receptor signaling pathway & $4.47 \times 10$ \\
\hline
\end{tabular}

GnRH, gonadotropin-releasing hormone; MAPK, mitogen associated protein kinase.

\section{Acknowledgements}

Not applicable.

\section{Funding}

The present study was supported by Public Projects of Zhejiang Province (grant no. GF18H040021).

\section{Availability of data and materials}

The datasets analyzed in the present study are all available on NCBI GEO (https://www.ncbi.nlm.nih.gov/geo/query/acc.cgi).

\section{Authors' contributions}

JW, SL and XL designed the study. JW and LW wrote the manuscript. JW, LW and YJ performed the bioinformatics analysis. All authors read and approved the final manuscript.

\section{Ethics approval and consent to participate}

Not applicable.

\section{Patient consent for publication}

Not applicable.

\section{Competing interests}

The authors declare that they have no competing interests.

\section{References}

1. Gimm O: Thyroid cancer. Cancer Lett 163:143-156, 2001.

2. Maia FF, Vassallo J, Pinto GA, Pavin EJ, Matos PS and ZantutWittmann DE: Expression of Mcl-1 and Ki-67 in papillary thyroid carcinomas. Exp Clin Endocrinol Diabetes 124: 209-214, 2016.

3. Wen Q, Zhao L, Wang T, Lv N, Cheng X, Zhang G and Bai L: LncRNA SNHG16 drives proliferation and invasion of papillary thyroid cancer through modulation of miR-497. Onco Targets Ther 12: 699-708, 2019.

4. Ambros V: The functions of animal microRNAs. Nature 431: 350-355, 2004.

5. Bartel DP: MicroRNAs: genomics, biogenesis, mechanism, and function. Cell 116: 281-297, 2004.

6. Feng B, Dong TT, Wang LL, Zhou HM, Zhao HC, Dong F and Zheng $\mathrm{MH}$ : Colorectal cancer migration and invasion initiated by microRNA-106a. PLoS One 7: e43452, 2012.

7. Zhu Z, Yang Q, Zhang B, Wu W, Yuan F and Zhu Z: miR-106b promotes metastasis of early gastric cancer by targeting ALEX1 in vitro and in vivo. Cell Physiol Biochem 52: 606-616, 2019. 
8. Yin Y, Hong S, Yu S, Huang Y, Chen S, Liu Y, Zhang Q, Li Y and Xiao H. MiR-195 inhibits tumor growth and metastasis in papillary thyroid carcinoma cell lines by targeting CCND1 and FGF2. Int J Endocrinol 2017: 6180425, 2017.

9. Wang D, Wang H, Li Y and Li Q. MiR-362-3p functions as a tumor suppressor through targeting MCM5 in cervical adenocarcinoma. Biosci Rep 38: pii: BSR20180668, 2018.

10. Park YR, Seo SY, Kim SL, Zhu SM, Chun S, Oh JM, Lee MR, Kim SH, Kim IH, Lee SO et al: MiRNA-206 suppresses PGE2-induced colorectal cancer cell proliferation, migration, and invasion by targetting TM4SF1. Biosci Rep 38, 2018. pii: BSR20180664.

11. Minna E, Romeo P, Dugo M, De Cecco L, Todoerti K, Pilotti S, Perrone F, Seregni E, Agnelli L, Neri A et al: miR-451a is underexpressed and targets AKT/mTOR pathway in papillary thyroid carcinoma. Oncotarget 7: 12731-12747, 2016.

12. Xia F, Wang W, Jiang B, Chen Y and Li X. DNA methylationmediated silencing of miR-204 is a potential prognostic marker for papillary thyroid carcinoma. Cancer Manag Res 11: $1249-1262,2019$

13. Chen $\mathrm{H}$ and Boutros PC: VennDiagram: A package for the generation of highly-customizable Venn and Euler diagrams in R. BMC Bioinformatics 12: 35, 2011.

14. Saiselet M, Gacquer D, Spinette A, Craciun L, DecaussinPetrucci M, Andry G, Detours V and Maenhaut C: New global analysis of the microRNA transcriptome of primary tumors and lymph node metastases of papillary thyroid cancer. BMC Genomics 16: 828, 2015.

15. Sticht C, De La Torre C, Parveen A and Gretz N: miRWalk: An online resource for prediction of microRNA binding sites. PLoS One 13: e0206239, 2018.

16. Shannon P, Markiel A, Ozier O, Baliga NS, Wang JT, Ramage D, Amin N, Schwikowski B and Ideker T: Cytoscape: A software environment for integrated models of biomolecular interaction networks. Genome Res 13: 2498-2504, 2003.
17. Yu G, Wang LG, Han Y and He QY: clusterProfiler: An R package for comparing biological themes among gene clusters. OMICS 16: 284-287, 2012

18. Gao X, Wang $\mathrm{J}$ and Zhang S: Integrated bioinformatics analysis of hub genes and pathways in anaplastic thyroid carcinomas. Int J Endocrinol 2019: 9651380, 2019.

19. Gao X, Wang X and Zhang S: Bioinformatics identification of crucial genes and pathways associated with hepatocellular carcinoma. Biosci Rep 38: pii: BSR20181441, 2018.

20. Zhang S, Wang Q, Han Q, Han H and Lu P: Identification and analysis of genes associated with papillary thyroid carcinoma by bioinformatics methods. Biosci Rep 39: pii: BSR20190083; 2019.

21. Jia M, Shi Y, Li Z, Lu X and Wang J: MicroRNA-146b-5p as an oncomiR promotes papillary thyroid carcinoma development by targeting CCDC6. Cancer Lett 443: 145-156, 2019.

22. Zhang XF, Ye Y, Zhao SJ. LncRNA Gas5 acts as a ceRNA to regulate PTEN expression by sponging miR-222-3p in papillary thyroid carcinoma. Oncotarget 9: 3519-3530, 2017.

23. Jiang L, Wu Z, Meng X, Chu X, Huang H and Xu C: LncRNA HOXA-AS2 facilitates tumorigenesis and progression of papillary thyroid cancer by modulating the miR-15a-5p/HOXA3 axis. Hum Gene Ther 30: 618-631, 2019.

24. Zhang H, Cai Y, Zheng L, Zhang Z, Lin X and Jiang N: LncRNA BISPR promotes the progression of thyroid papillary carcinoma by regulating miR-21-5p. Int J Immunopathol Pharmacol 32: 2058738418772652, 2018. https://doi. org $/ 10.1177 / 2058738418772652$.

25. Swierniak M, Wojcicka A, Czetwertynska M, Stachlewska E, Maciag M, Wiechno W, Gornicka B, Bogdanska M, Koperski L, de la Chapelle A et al: In-depth characterization of the microRNA transcriptome in normal thyroid and papillary thyroid carcinoma. J Clin Endocrinol Metab 98: E1401-1409, 2013.

26. Chen S, Fan X, Gu H, Zhang L and Zhao W: Competing endogenous RNA regulatory network in papillary thyroid carcinoma. Mol Med Rep 18: 695-704, 2018. 